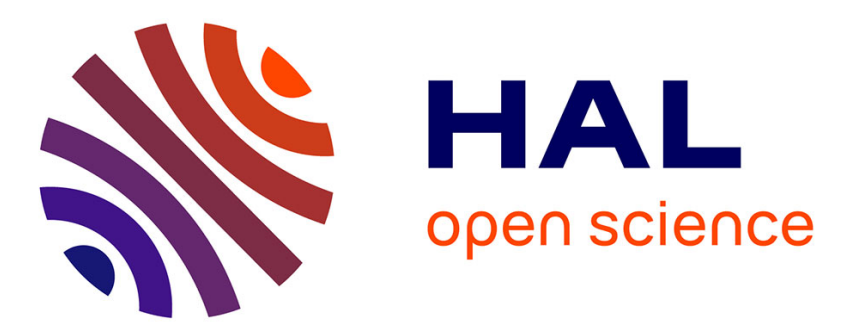

\title{
The effect of surface temperature on optical properties of molybdenum mirrors in the visible and near-infrared domains
}

\author{
Marco Minissale, G.T. Zeweldi, Régis Bisson, Laurent Gallais
}

\section{- To cite this version:}

Marco Minissale, G.T. Zeweldi, Régis Bisson, Laurent Gallais. The effect of surface temperature on optical properties of molybdenum mirrors in the visible and near-infrared domains. Nuclear Fusion, 2018, 58 (9), pp.096012. 10.1088/1741-4326/aaca03 . hal-01953124

HAL Id: hal-01953124

https://hal-amu.archives-ouvertes.fr/hal-01953124

Submitted on 13 Dec 2018

HAL is a multi-disciplinary open access archive for the deposit and dissemination of scientific research documents, whether they are published or not. The documents may come from teaching and research institutions in France or abroad, or from public or private research centers.
L'archive ouverte pluridisciplinaire HAL, est destinée au dépôt et à la diffusion de documents scientifiques de niveau recherche, publiés ou non, émanant des établissements d'enseignement et de recherche français ou étrangers, des laboratoires publics ou privés.

\section{(c)(1)}

Distributed under a Creative Commons Attribution| 4.0 International License 


\title{
The effect of surface temperature on optical properties of molybdenum mirrors in the visible and near-infrared domains
}

\author{
Marco Minissale ${ }^{a, b}$, Gebrehiwot Tesfay Zeweldi ${ }^{b}$, Régis Bisson ${ }^{a}$, \\ Laurent Gallais ${ }^{b}$ \\ ${ }^{a}$ Aix Marseille Univ, CNRS, PIIM, Marseille, France \\ ${ }^{b}$ Aix Marseille Univ, CRNS, Centrale Marseille, Institut Fresnel, Marseille, France \\ E-mail: marco.minissale@univ-amu.fr \\ DOI : https://doi.org/10.1088/1741-4326/aaca03 \\ Nucl.Fusion 58 (2018) 096012
}

\begin{abstract}
Molybdenum mirrors will be used in several optical diagnostics to control the plasma in the ITER tokamak. In this harsh environment, mirrors can undergo transient temperature rises. Thus the knowledge of the temperature dependence of optical properties of molybdenum is necessary for a good operation of optical systems in ITER.

Molybdenum optical properties have been extensively studied at room temperature, but little has been done at high temperatures in the visible and near-infrared domains. We investigate here the temperature dependence of molybdenum reflectivity from the ambient to high temperatures $(<800 \mathrm{~K})$ in the $500-1050 \mathrm{~nm}$ spectral range. Experimental measurements of reflectivity, performed via a spectroscopic system coupled with laser remote heating, show a maximum increase of $2.5 \%$ at $800 \mathrm{~K}$ in the 850-900 $\mathrm{nm}$ wavelength range and a non-linear temperature dependency as a function of wavelength.

We describe these dependencies through a Fresnel and a Lorentz-Drude model. The Fresnel model accurately reproduces the experimental curve at a given temperature by using a parabolic temperature dependency for the refractive index, $n$, and a linear dependency for the extinction coefficient, $k$.

We develop a Lorentz-Drude model to describe the interaction of light with charge carriers in the solid. This model includes temperature dependency on both intraband (Drude) and interband (Lorentz) transitions. It is able to reproduce the experimental results quantitatively, highlighting a non-trivial dependency of interband transitions on temperature. Eventually, we use the Lorentz-Drude model to evaluate the total emissivity of molybdenum from $300 \mathrm{~K}$ to $2800 \mathrm{~K}$, and we compare our experimental and theoretical findings with previous results.
\end{abstract}

Keywords: ITER, First Mirrors, Reflectivity, Temperature, Molybdenum, LorentzDrude model, Refractive index, Total emissivity 


\section{Introduction}

Many spectroscopic techniques will be used in the ITER tokamak to control the plasma, both to prevent damage to the first wall and to improve the tokamak performance. Such optical diagnostic systems will work in the 0.05-1000 nm wavelength range [1]. In particular, visible and near-infrared regions will be of interest for three main diagnostics:

- ITER LIDAR (LIght Detection And Ranging): actively cooled mirrors will be used to guide the high power laser beam, whose working wavelength is $800 \mathrm{~nm}$.

- $\mathrm{H}_{\text {alpha }}$ spectroscopy: the system measures Balmer lines (transitions of principal quantum number from $n=3-8$ to $n=2$ ) emitted from hydrogen isotopes in the wavelength range $370-660 \mathrm{~nm}$.

- Divertor impurity monitor(s) or core and edge charge-exchange diagnostics: identification of the impurity species arriving at the divertor targets and measurement of their distribution and fluxes will be made by spectroscopic measurements in the wavelength range 200-1000 $\mathrm{nm}$.

The first element of these optical diagnostic systems in ITER will be metallic mirrors (i.e. molybdenum and rhodium) called First Mirrors (FMs). FMs have to operate in extreme conditions since they will be exposed to intense UV and x-ray radiation ( up to $\left.500 \mathrm{~kW} / \mathrm{m}^{2}\right)$, neutron heating ( up to $\left.8 \mathrm{~W} / \mathrm{cm}^{3}\right), \gamma$ rays, and particle fluxes arising from charge exchange atoms (CXA) up to $2 \cdot 10^{19}$ particles $\mathrm{m}^{-2} \mathrm{~s}^{-1}$ with energies up to several keV [2]. In this harsh environment, FMs must maintain their optical properties to be used as reliable tool to control plasma parameters [3-5]. A change of FMs reflectivity can lead to a failure of the related diagnostic systems and a consequent impact on the reactor operability. For these reasons, it is highly important to know how ITER environment can affect FMs reflectivity due to erosion and/or impurity deposition. It has been shown that light impurities such as Be or $\mathrm{C}$ can form thin coating on mirror surfaces [6-9]. Such coatings will determine the reflectivity of the mirrors more than anything else. Several works have characterized the degradation of the optical reflectivity of FMs after exposure in a tokamak [10] and as a result of the interactions with edge plasma, ions or neutrons [11-13]. Recently, some experimental works have proposed different methods to recover FM reflectivity by cleaning mirrors in situ through interaction with plasma and ions [14-18].

Structural modifications of metal surface, due to sputtering and impurity deposition, induce strong reflectivity variations (up to 55\% [19]); nevertheless other phenomena can cause considerable variations of metal reflectivity. As discussed by Salewski et al. [20], under ITER photon/particle fluxes FMs can reach temperatures of $500 \mathrm{~K}$ and $800 \mathrm{~K}$ in presence of active or radiation cooling, respectively. We have already shown in a previous work that a temperature increase of $600 \mathrm{~K}$ induces a reflectivity variation of tungsten up to $9 \%$ at $850 \mathrm{~nm}$ [21]. In this work, we present the first temperature dependent measurements of molybdenum (Mo) reflectivity in the optical and near infrared (NIR) wavelength range $(500-1050 \mathrm{~nm})$ in the $300-780 \mathrm{~K}$ temperature 
range, results of interest for ITER FMs used in the diagnostics listed above. However our experimental measurements cannot be applied to other important diagnostics, like the IR section of the Wide Angle Viewing System operating at much longer wavelengths of 3-5 micrometer and used e.g. for measurements of temperature of plasma facing components. Nevertheless we stress that the same methodology could be used to extend our work in the MIR using a MIR supercontinuum source and spectrometer. We also provide a quantitative determination of the refractive index and the extinction coefficient of Mo, and their temperature dependencies, by using Fresnel equations. Subsequently, we describe a Lorentz-Drude model to study the temperature dependence of free carriers and optical phonons in Mo.

\section{Experimental setup}

The experiments were conducted with the experimental setup described previously by Minissale et al. [22]. It consists in a vacuum chamber (base pressure $5 \cdot 10^{-3} \mathrm{mbar}$ ) where is located a sample holder made in Macor. The polycrystalline molybdenum sample was provided by II-VI Deutschland GmbH with a mirror-like polishing. We have evaluated the roughness at the center of the sample (an area of $1 \mathrm{~mm}^{2}$ corresponding to the supercontinuum beam diameter) through an optical profilometer (Zygo Newview) and we found $\mathrm{R}_{a} \sim 1-2 \mathrm{~nm}$.

The vacuum chamber is provided with two optical ports made of $\mathrm{BaF}_{2}$ and fused silica windows. The $\mathrm{BaF}_{2}$ window is used to heat the sample via a continuous wave high-power laser emitting at $805 \mathrm{~nm}$ (JENOPTIK Laser GmbH) and to control the temperature exsitu through an infrared camera (FLIR, model A655sc) working in the 7.5-14 $\mu \mathrm{m}$ spectral range. Additionally, the sample temperature is checked in situ with a thermocouple (type-K).

The fused silica window is used to perform spectroscopic measurements in the 500$1050 \mathrm{~nm}$ range through the combination of a supercontinuum laser beam, an integrating sphere and a fiber-coupled spectrometer.

The Mo reflectivity is determined by using the following equation

$$
R_{\text {Mo }}=\frac{S_{\text {meas }}-S_{\text {Dark }}}{S_{\text {ref }}-S_{\text {Dark }}} \frac{1}{T_{\text {Siw }}^{2}}
$$

where $\mathrm{S}_{\text {meas }}$ and $\mathrm{S}_{\text {ref }}$ are the spectra measured by sending the supercontinuum beam on the Mo sample and to a reference silver mirror, respectively; $\mathrm{S}_{\text {Dark }}$ is the dark spectrum obtained with the supercontinuum beam switched off and the sample heated to the desired temperature; $\mathrm{T}_{\text {Siw }}$ represents the transmission of the supercontinuum beam through the fused silica window.

Reflectivity has been studied by varying three parameters:

- Polarization of the supercontinuum beam. Parallel $(\mathrm{P})$ or perpendicular $(\mathrm{S})$ to the plane of incidence. 


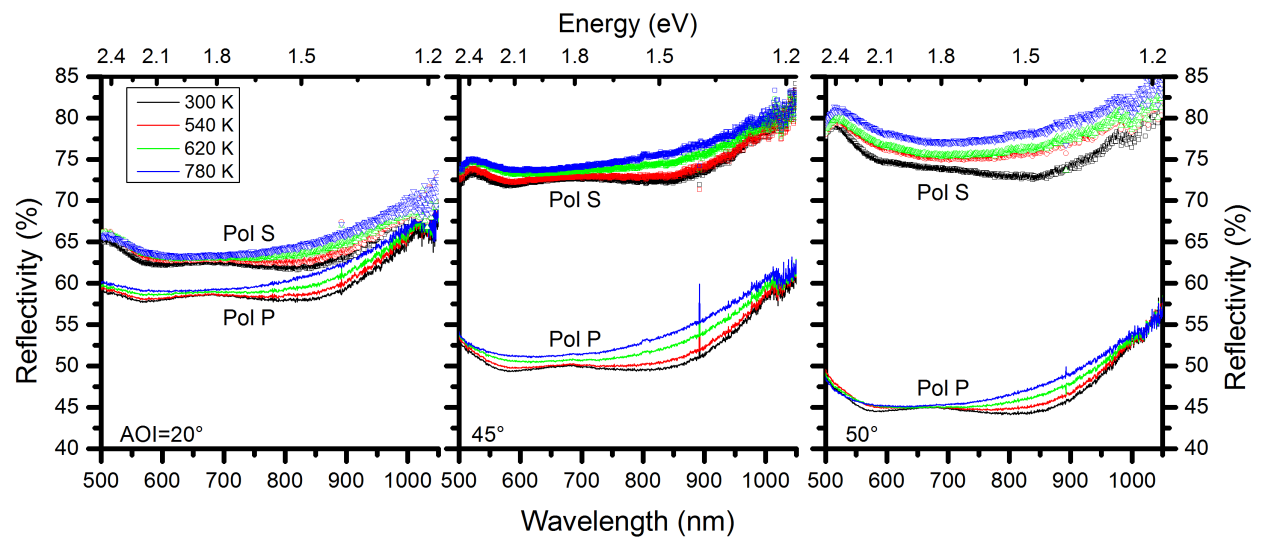

Figure 1. Reflectivity for molybdenum of S (dots) and P-polarized (lines) light at four different temperatures $(300,540,620$, and $780 \mathrm{~K}$ ) for three incidence angles (from left to right, 20,45 , and $50^{\circ}$ ) as a function of wavelength.

- Sample temperature. Temperature is varied between 300 and $780 \mathrm{~K}$.

- Angle of incidence (AOI) of the supercontinuum beam. We have used three angles of incidence with respect to the surface normal: $20^{\circ}, 45^{\circ}, 50^{\circ}$.

\section{Experimental results}

We present in Fig. 1 the spectral reflectivity of the molybdenum sample held at different temperatures $(300 \pm 3,540 \pm 14,620 \pm 15$, and $780 \pm 20 \mathrm{~K})$ for $\mathrm{S}$ and $\mathrm{P}$ polarizations. We have studied molybdenum reflectivity for three AOIs of light: $20^{\circ}, 45^{\circ}$, and $50^{\circ}$ (from the left to the right in Figure 1). Table 1 sums up some of the results discussed in this section. In particular we list the reflectivity of unpolarized light obtained through the following equation

$$
R_{\text {non-pol }}=\frac{R_{s}+R_{p}}{2}
$$

as a function of temperature and wavelength for $\mathrm{AOI}=20^{\circ}$.

At first glance, one can see that an increase of the sample temperature produces an increase of the surface reflectivity. This growth is not linear both as a function of wavelength and temperature. In the left panel of Fig. 2, we present the variation (with respect to room temperature) of reflectivity as a function of wavelength in the case of P-polarized light $\left(\mathrm{AOI}=20^{\circ}\right)$. The highest variation is at $900 \mathrm{~nm}$ where reflectivity goes from $59.4 \pm 0.1 \%$ at $300 \mathrm{~K}$ to $61.6 \pm 0.3 \%$ at $780 \mathrm{~K}$ that means a percentage increase higher than $\sim 4.2 \pm 0.5 \%$, as shown in the right panel of Fig. 2 . We note that between 650 and $700 \mathrm{~nm}$, sample temperature has a weaker influence on the reflectivity with percentage increases smaller than $1 \pm 0.5 \%$. For all other wavelengths, we have measured relative variations comprised between 1 and $4 \%$. Similar behaviors have been observed also for other AOIs and for S-polarized light. As a general comment, we note that the temperature dependence of reflectivity is not constant as a function of wavelength. 
Table 1. Temperature dependence of Mo reflectivity for different wavelengths.

\begin{tabular}{cccccc}
\hline \hline$\lambda$ & Energy & \multicolumn{4}{c}{ Reflectivity $^{a}$} \\
$\mathrm{~nm}$ & $\mathrm{eV}$ & $300 \mathrm{~K}$ & $540 \mathrm{~K}$ & $620 \mathrm{~K}$ & $780 \mathrm{~K}$ \\
\hline 500 & 2.25 & $62.1^{b}$ & 62.7 & 62.9 & 62.7 \\
550 & 2.25 & 60.7 & 61.7 & 61.7 & 61.7 \\
600 & 2.07 & 60.1 & 60.5 & 60.9 & 61.1 \\
650 & 1.91 & 60.5 & 60.7 & 61.0 & 61.1 \\
700 & 1.77 & 60.4 & 60.7 & 60.9 & 61.1 \\
750 & 1.65 & 60.1 & 60.4 & 60.9 & 61.3 \\
800 & 1.55 & 59.9 & 60.5 & 61.4 & 62.0 \\
850 & 1.46 & 60.1 & 60.9 & 61.8 & 62.6 \\
900 & 1.38 & 61.1 & 61.9 & 62.9 & 63.7 \\
950 & 1.31 & 63.6 & 64.4 & 65.1 & 65.7 \\
1000 & 1.24 & 66.7 & 67.1 & 67.4 & 67.8 \\
1050 & 1.18 & 69.7 & 69.6 & 69.7 & 69.8 \\
\hline \hline
\end{tabular}

${ }^{a}$ Reflectivity (in \%) of unpolarized light at $\mathrm{AOI}=20^{\circ}$ obtained as a mean between $\mathrm{S}$ and $\mathrm{P}$-polarized spectra.

$b$ The uncertainty is comprised between 0.1 and 0.3 for all measurements at $\mathrm{AOI}=20^{\circ}$.
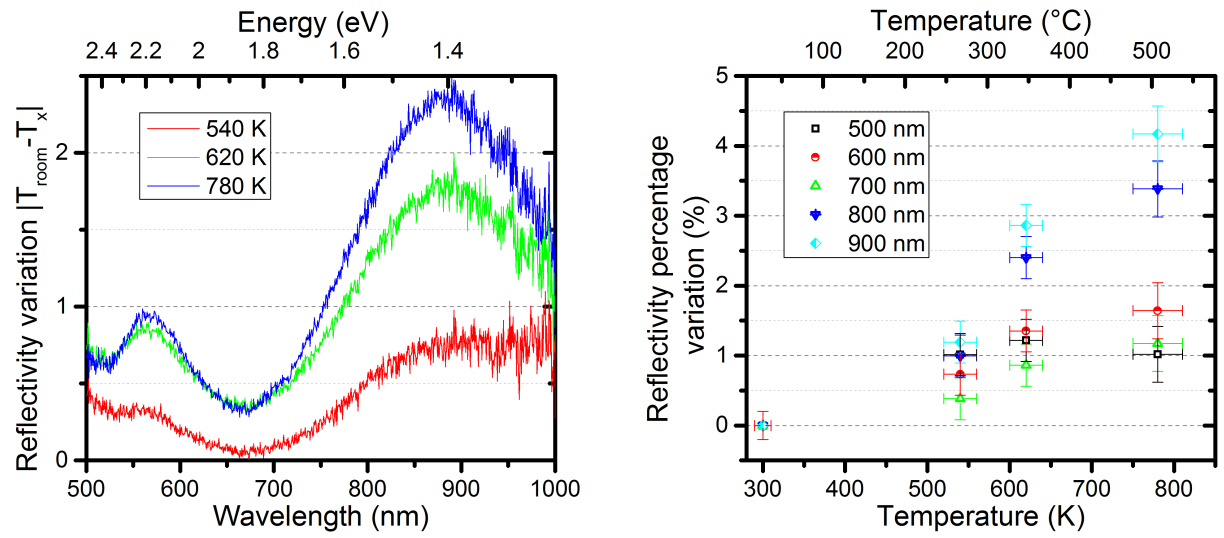

Figure 2. Left panel: reflectivity variation (from the $300 \mathrm{~K}$ value) of molybdenum for P-polarized light $\left(\mathrm{AOI}=20^{\circ}\right)$ at three temperatures $\mathrm{T}_{x}(540,620$, and $780 \mathrm{~K})$ as a function of wavelength. Right panel: Reflectivity of molybdenum for P-polarized light $\left(\mathrm{AOI}=20^{\circ}\right)$ at five wavelengths $(500,600,700,800$, and $900 \mathrm{~nm})$ as a function of temperature.

In particular the most evident effect of the temperature increase is to "flatten" the reflectivity spectrum. This effect has been already observed in the case of tungsten [21] and it has been explained through a different response of each Lorentz oscillators of the material to a temperature increase. 
Table 2. Refractive index, $n$, and extinction coefficient, $k$, of molybdenum at four different temperatures from visible to near infrared spectral range.

\begin{tabular}{|c|c|c|c|c|c|c|c|c|}
\hline \multirow[b]{2}{*}{$\begin{array}{c}\lambda \\
(n m)\end{array}$} & \multicolumn{8}{|c|}{ Temperature (K) } \\
\hline & \multicolumn{4}{|c|}{$n$} & \multicolumn{3}{|c|}{$k$} & 780 \\
\hline 500 & 3.07 & 3.11 & 3.05 & 3.11 & 3.94 & 4.02 & 4.13 & 4.28 \\
\hline 550 & 3.06 & 3. & 3.20 & 3.26 & 3.70 & 3.83 & 3 & 4.08 \\
\hline 600 & 3.10 & 3.27 & 3.29 & 3.35 & 3.68 & 3.86 & 3. & 4.12 \\
\hline 650 & 3.17 & 3.36 & 3.35 & 3.42 & 3.70 & 3.93 & 4.00 & 4.19 \\
\hline 700 & 3.23 & 3.4 & 3.41 & 3.47 & 3.69 & 3.96 & 4.03 & 4.22 \\
\hline 750 & 3.27 & 3.48 & 3.47 & 3.53 & 3.65 & 3.95 & 4.02 & 4.20 \\
\hline 800 & 3.25 & 3.50 & 3.52 & 3.58 & 3.63 & 3.94 & 4.03 & 4.19 \\
\hline 850 & 3.19 & 3.48 & 3.56 & 3.62 & 3.68 & 3.99 & 4.09 & 4.24 \\
\hline 900 & 3.08 & 3.42 & 3.55 & 3.61 & 3.87 & 4.15 & 4.26 & 4.40 \\
\hline 950 & 2.93 & 3.31 & 3.47 & 3.53 & 4.23 & 4.40 & 4.51 & 4.66 \\
\hline 1000 & 2.75 & 3.15 & 3.26 & 3.32 & 4.69 & 4.67 & 4.76 & 4.93 \\
\hline 1050 & 2.58 & 2.91 & 2.87 & 2.93 & 5.13 & 4.75 & 4.80 & 5.01 \\
\hline
\end{tabular}

\section{Models}

\subsection{Fresnel model: evaluation of $n$ and $k$}

The refractive index, $n$, and extinction coefficient, $k$, of bulk metallic molybdenum were determined by fitting the theoretical reflectivities numerically to the corresponding experimental values. A model, based on Fresnel reflectance equations, has been developed for this purpose which takes a set of $n$ and $k$ values as an input and generates all possible combinations of elements from the two sets. We stress that we have imposed an initial guess for $n$ and $k$ in order to optimize the algorithm and to reduce the calculation time. For each wavelength, from $500 \mathrm{~nm}$ to $1050 \mathrm{~nm}$ with a step of 10 $\mathrm{nm}$, we have used as initial guess for $n$ at room temperature the mean $\bar{n}$ estimated from four previous works [23-26] dealing with molybdenum reflectivity. To test all values of refractive index from previous works we have varied $n$ in the following domain

$$
\left(\bar{n}(\lambda)-1.5 \cdot \sigma_{\bar{n}(\lambda)}\right)<n(\lambda)<\left(\bar{n}(\lambda)+1.5 \cdot \sigma_{\bar{n}(\lambda)}\right)
$$

where $\sigma_{\bar{n}(\lambda)}$ is the population standard deviation. A similar approach has been used for $k$ both for the initial guess and for the domain at room temperature. The best-fit of experimental data has been found varying $\mathrm{n}$ and $\mathrm{k}$ in the given wavelength domain and minimizing the Pearson chi square weighted through the error of experimental data $(\sigma)$ as described in [21].

The values of $n(\lambda)$ and $k(\lambda)$ determined at $300 \mathrm{~K}$ have been used as initial guesses for $n(\lambda)$ and $k(\lambda)$ at $540 \mathrm{~K}$; these values, in turn, have been used for $n(\lambda)$ and $k(\lambda)$ at 620 $\mathrm{K}$ and so on, until the highest temperature. With the exception of values determined at $300 \mathrm{~K}$, the sweeping range has been arbitrarily set to $1( \pm 0.5)$ both for $n$ and $k$. The values of $n$ and $k$ giving the best fit between theoretical and experimental reflectivities were determined by a multiple iterations routine. Table 2 shows the evaluated $n$ and $k$ values of molybdenum at four different sample temperatures. The reflectivity calculated using these $n$ and $k$ values gives a good match with the measured reflectivity as shown in Fig. 3(a) for the case of $\lambda=950 \mathrm{~nm}$. Similar results have been obtained for all 

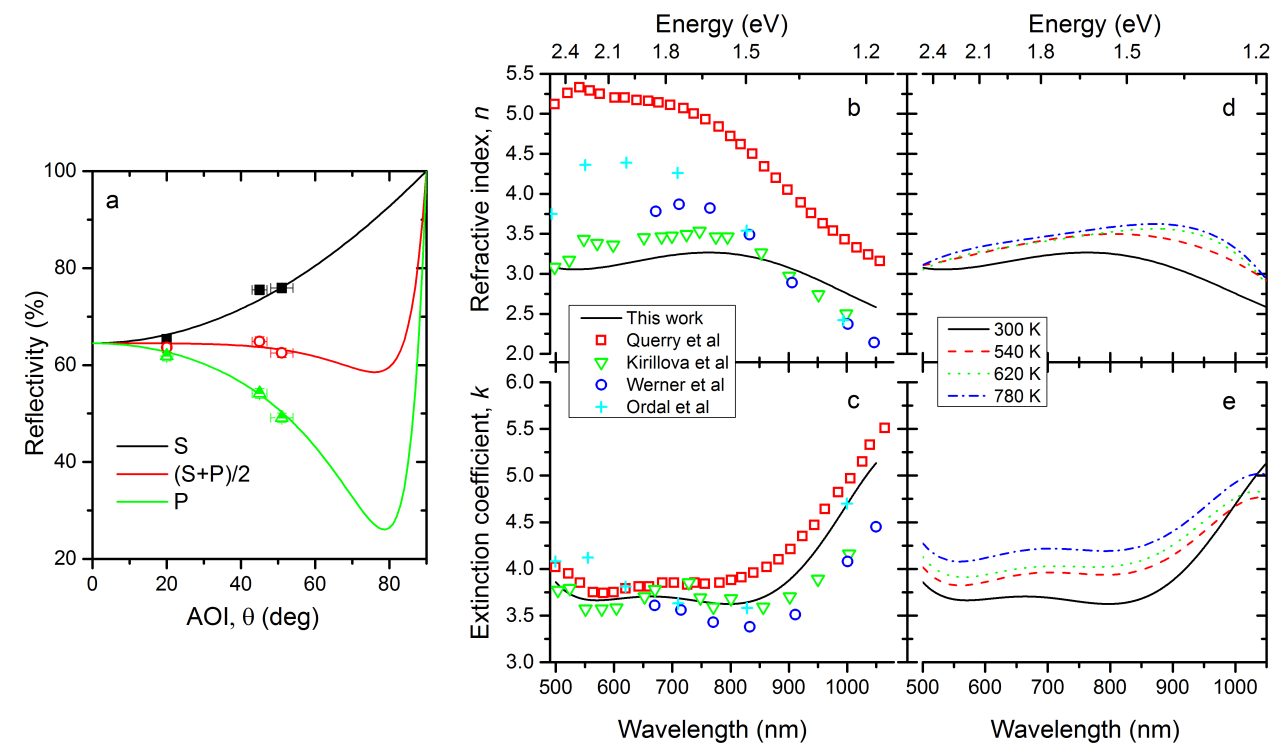

Figure 3. Panel $a$. Reflectivity versus angle of incidence of molybdenum at $300 \mathrm{~K}$ for $\mathrm{S}, \mathrm{P}$ and unpolarized light at $950 \mathrm{~nm}$ : dots-experimental data, lines-theoretical; Panels $b$ and $c$. Refractive index $n$ and extinction coefficient $k$ of molybdenum at $300 \mathrm{~K}$ as a function of wavelength calculated through a Fresnel model (lines). Dots are taken from Querry et al. [23] (squares), Werner et al. [24] (crosses), Kirillova et al. [25] (triangles), Ordal et al. [26] (circles); Panels $d$ and $e$. Refractive index $n$ and extinction coefficient $k$ of molybdenum as a function of wavelength for different temperatures: 300, 540, 620 , and $780 \mathrm{~K}$.

wavelengths. As previously said, some works have studied $n$ and $k$ of molybdenum at room temperature [23-26] while no data are present at high sample temperatures. The $n$ values found in literature are quite dispersed in the 500-1050 nm range (see Fig. 3(b)); for example Kirillova et al. [25] and Querry et al. [23] provide values respectively of 3 and 5 at $500 \mathrm{~nm}$. Our estimate of $n$ seems to follow Kirillova et al. with a maximum deviation of $11 \%$ from its values at 550 and $1000 \mathrm{~nm}$. On the contrary, $k$ values are quantitatively similar to those reported in previous works. Even in this case, our values are in good agreement with Kirillova et al., with a deviation lower than $4 \%$ in the $500-950 \mathrm{~nm}$ range. Our results underestimate Querry's values with deviations ranging from $0.1 \%$ to $7 \%$ (at $840 \mathrm{~nm}$ ) (see Fig. 3(c)).

From Figs. 3(d) and (e), one can note that the temperature evolution of $n$ and $k$ is both a function of temperature and wavelength. Nevertheless, by restricting the spectral domain to 500-950 nm, temperature and wavelength variables can be decoupled and $k$ can be described through a linear temperature dependence

$$
k(\lambda, T)=k(\lambda)_{300 K}+9.5(2.3) \cdot 10^{-4} \cdot T
$$

where in parenthesis we give the standard deviation calculated using Table 2 data. By 
using the same approach we find a parabolic temperature dependence for $n$

$$
\begin{aligned}
n(\lambda, T)= & n(\lambda)_{300 K}+1.96(1.13) \cdot 10^{-3} \cdot T+ \\
& +1.17(0.73) \cdot 10^{-6} \cdot T^{2}
\end{aligned}
$$

We pinpoint that in this case the decoupling is more complicated and higher values of the standard deviation have been found.

\subsection{Lorentz-Drude model}

We have developed a classical Lorentz-Drude (LD) model to simulate the electric permittivity of metals and to calculate molybdenum reflectivity. This model, based on the formalism presented in [21], treats free (Drude term) and harmonically bound (Lorentz terms) electrons, as damped particles subjected to external electric fields. The model is described by the following equation:

$$
\begin{gathered}
\hat{\epsilon}_{r}(T)=1-\frac{f_{0} \omega_{p}(T)^{2}}{\omega\left(\omega-i \Gamma_{D}(T)\right)}+ \\
\sum_{i=1}^{4} \frac{f_{i} \omega_{p}(T)^{2}}{\omega_{i}(T)^{2}-\omega^{2}+i \omega \Gamma_{i}(T)}
\end{gathered}
$$

where $f_{0}$ and $\Gamma_{D}$ are the strength and the damping of the Drude oscillator, while $f_{i}$, $\omega_{i}$, and $\Gamma_{i}$ are the strength, the frequency, and the damping of the Lorentz oscillators, respectively. The temperature dependence is included in the damping factors and in the resonance frequencies. In particular for Drude damping we use

$$
\begin{aligned}
& \Gamma_{D}(T)=\Gamma_{e-p h}(T)+\Gamma_{e-e}(T)= \\
& \Gamma_{0}\left(\frac{2}{5}+4\left(\frac{T}{\theta}\right)^{5} \int_{0}^{\theta / T} \frac{z^{4} d z}{e^{z}-1}\right)+ \\
& +\frac{\pi^{3}}{12 \hbar E_{f}}\left[\left(k_{b} T\right)^{2}+(\hbar \omega)^{2}\right]
\end{aligned}
$$

where $\Gamma_{e-e}$ and $\Gamma_{e-p h}$ are the electron-electron and electron-phonon damping, respectively, $\theta$ is the Debye temperature of molybdenum $(380 \mathrm{~K}), \mathrm{E}_{f}$ is the Fermi energy of molybdenum $(5.867 \mathrm{eV}), \Gamma_{0}$ is the damping coefficient of electron-phonon term, $k_{b}$ is the Boltzmann constant and $\hbar$ is the Planck constant.

We stress that in the $500-1050 \mathrm{~nm}$ the reflectivity is only slightly influenced by the temperature dependence of intraband term. For such reason, we added the temperature dependence on Lorentz oscillators (interband terms), too. For Lorentz terms ( $i$ from 1 to 4 ), the damping is described by

$$
\Gamma_{i}(T)=\Gamma_{i}+\alpha\left(\sqrt{T}-\sqrt{T_{\text {room }}}\right)
$$

where $\alpha$ is a parameter expressed in $\mathrm{eV} \cdot K^{-1 / 2}$. 
The effect of surface temperature on optical properties of molybdenum mirrors

Table 3. Values of the Lorentz-Drude parameters for molybdenum.

\begin{tabular}{lllll}
\hline \hline Oscillator & $i$ & $\omega_{i}$ & $\mathrm{f}_{i}$ & $\Gamma_{i}$ \\
\hline Drude & 0 & 0 & 0.72 & 0.02 \\
Lorentz & 1 & 1.71 & 0.12 & 0.74 \\
Lorentz & 2 & 2.48 & 0.54 & 1.45 \\
Lorentz & 3 & 3.19 & 1.72 & 7.34 \\
Lorentz & 4 & 7.94 & 3.03 & 7.52 \\
\hline \hline & $\omega_{i}$ and $\Gamma_{i}$ are given in $\mathrm{eV}$, \\
$\mathrm{f}_{i}$ are dimensionless.
\end{tabular}

The temperature dependence of the plasma frequency includes a reduced electronic density due to the thermal volume expansion [27] and it is given by

$$
\omega_{p}(T)=\frac{\omega_{p}}{\sqrt{1+\gamma\left(T-T_{\text {room }}\right)}},
$$

while the resonance frequency of Lorentz oscillators can be written as follows

$$
\omega_{i}(T)=\sqrt{\omega_{i}^{2}-\beta\left(\sqrt{T}-\sqrt{T_{\text {room }}}\right)}
$$

where $\beta$ is a parameter expressed in $e V^{2} \cdot K^{-1 / 2}$.

To the best of our knowledge, the LD model has never been used to describe the electric permittivity of molybdenum and a lack of theoretical data is present in literature. To constrain the initial guess of LD parameters $\left(\Gamma_{i}, \omega_{i}\right.$, and $\left.\mathrm{f}_{i}\right)$, we have started from parameters of tungsten [21], since these metals present similar features in the considered wavelength range. The parameters shown in Table 3 have been found by minimizing the Pearson's $\chi^{2}$ value between measured and calculated reflectivity at room temperature. This model is able to fit quantitatively experimental results at $300 \mathrm{~K}$. The deviation between model and experiments at room temperature is lower than $1.2 \%$ if we consider the 500-1000 nm region, while it is lower than $0.3 \%$ if we limit our analysis to the 500-950 nm region. We cannot find such good match between model and experiments at higher temperatures, when we set $\alpha$ and $\beta$ values to zero in Eqs 8 and 10. The model predicts the increase of reflectivity as a function of temperature but succeeds to fit only qualitatively experimental results. In order to improve our model we have used $\alpha$ and $\beta$ as free parameters by following [21]. By setting $\alpha=1.45 \cdot 10^{-2} \mathrm{eV} \cdot \mathrm{K}^{1 / 2}$. and $\beta=1.19 \cdot 10^{-3} \mathrm{eV}^{2} \cdot \mathrm{K}^{1 / 2}$ we are able to reduce the deviation to $\pm 1 \%$ as shown in the

right panel of Fig. 4. The comparison between calculated and experimental reflectivity (obtained through eq. 2 for unpolarized light at $20^{\circ}$ of AOI) as a function of wavelength is shown in the left panel of Fig. 4.

\subsection{Spectral and total emissivity}

The LD model presented in the previous section is used here to evaluate the spectral $\left(\epsilon_{\lambda}\right)$ and total emissivity $\left(\epsilon_{t o t}\right)$ of molybdenum. $\epsilon_{\lambda}$ is calculated through

$$
\epsilon_{\lambda}=1-R(\lambda)
$$



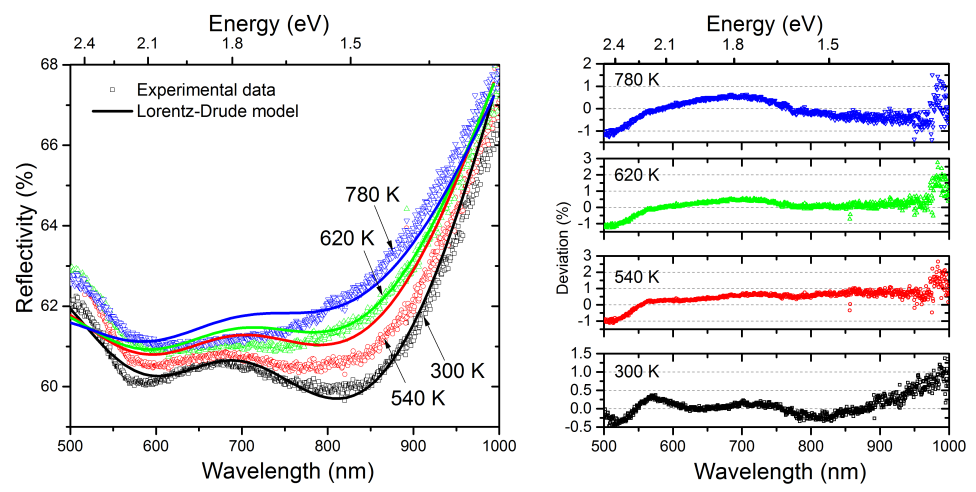

Figure 4. Left panel: measured and calculated reflectivity of unpolarized light at $20^{\circ}$ of AOI. Lines are obtained by using the Lorentz-Drude model for four different temperatures $(300,540,620$, and $780 \mathrm{~K})$. Right panel: deviation between experimental and calculated reflectivity.

valid for an opaque material (i.e. transmission=0). The total emissivity is subsequently estimated through the following equation

$$
\epsilon_{t o t}=\frac{\int_{\lambda_{1}}^{\lambda_{2}} \epsilon_{\lambda} \lambda^{-5} \cdot \exp \left[\frac{c 2}{\lambda T}\right]^{-1} d \lambda}{\int_{\lambda_{1}}^{\lambda_{2}} \lambda^{-5} \cdot \exp \left[\frac{c 2}{\lambda T}\right]^{-1} d \lambda}
$$

where $\mathrm{c} 2$ is the Planck's second radiation constant, $\lambda_{1}$ and $\lambda_{2}$ are the limits of integration. We stress that LD parameters have been optimized to fit reflectivity measurements between 300 and $800 \mathrm{~K}$ (in the 500-1050 nm range) and thus the LD model could lead to inaccurate results in a larger temperature range. Nevertheless we have broadened the temperature range up to $2800 \mathrm{~K}$ in order to overlap and compare our results to studies performed in the literature at high temperatures. Figure 5a shows the spectral emissivity of molybdenum in the $0.5-100 \mu \mathrm{m}$ region for different temperatures (from 300 to $1500 \mathrm{~K}) . \epsilon_{\lambda}$ decreases in the visible and NIR spectral regions (i.e. $0.5-1.1 \mu \mathrm{m}$ ) as a function of temperature (zoom in Fig. 5a), while it increases in the infrared (IR) domain. This behavior has been observed already by Cagran et al. [28], even if our $\epsilon_{\lambda}$ values match only qualitatively Cagran's ones; actually for the same temperature $\epsilon_{\lambda}$ from Cagran et al. is two times higher than values estimated with the LD model. This is more evident from Fig. 5b where we plot the temperature dependence of $\epsilon_{\lambda}$. In the 300$1500 \mathrm{~K}$ region LD model at $0.65 \mu \mathrm{m}$ match with the slight decrease of $\epsilon_{0.65 \mu m}$ measured at higher temperatures by Wall et al. [29]. The model starts to fail for temperature higher than $1500 \mathrm{~K}$ since it predicts an increase of emissivity up to 0.45 at 0.65 , 1 , and $2 \mu \mathrm{m}$ and to 0.3 at $10 \mu \mathrm{m}$. Such increase of $\epsilon_{\lambda}$ in the IR domain has a dramatic effect when LD model is used to calculate $\epsilon_{\text {tot }}$ (Eq. 12). The result is shown by solid line in Fig. 5c. We have evaluated $\epsilon_{\text {tot }}$ by using $\lambda_{1}$ and $\lambda_{2}$ equal to 0.5 and $100 \mu \mathrm{m}$, respectively. The LD model predicts an increase for $\epsilon_{\text {tot }}$ from 0.021 to almost 0.5 between 300 and 2800 K. Our results overlap with those of Rudkin et al. [32] and Abbott et al. [33] only between 1250 and $1500 \mathrm{~K}$ as shown in Fig. 5c and Table 4, but they differ for 

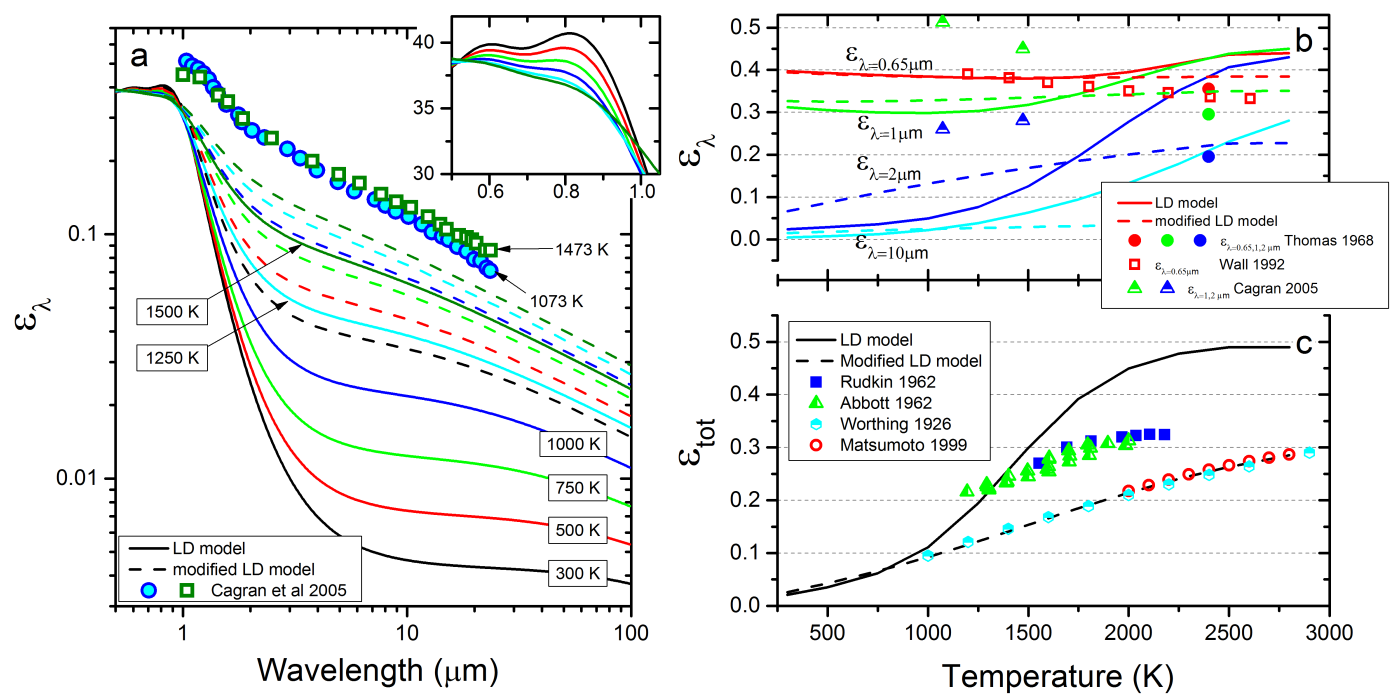

Figure 5. Panel a: Calculated (lines) and measured (symbols) spectral emissivity of tungsten as function of wavelength. Solid and dashed lines are obtained by using the standard (eq. 6 to 10) and the modified (eq. 13) LD models, respectively, for six different temperatures $(300,500,750,1000,1250$, and $1500 \mathrm{~K})$. Symbols are taken from Cagran et al. [28] (circles at $1073 \mathrm{~K}$ and squares at $1473 \mathrm{~K}$ ). Panel b: Calculated and measured spectral emissivity of tungsten as function of temperature. Solid and dashed lines are obtained by using the Lorentz-Drude models for four wavelengths $(0.65,1,2$, and $10 \mu \mathrm{m}$ ). Red symbols are taken from Wall et al. [29] (squares) and Thomas [30] (circles). Panel $c$ : Calculated or measured total emissivity of tungsten as function of temperature. Solid and dashed lines are obtained by using the Lorentz-Drude models. Symbols are taken from Matsumoto et al. [31] (circles), Rudkin et al. [32] (squares) and Abbott et al. [33] (triangles).

higher temperatures. The overview of $\epsilon_{\text {tot }}$ measurements presented in Matsumoto et al. [31] shows two distinct families of molybdenum samples shifted of $\Delta \epsilon_{\text {tot }} \sim 0.08$ with a quasi-linear temperature dependence of $\epsilon_{\text {tot }}$ at high temperature (above $1000 \mathrm{~K}$ ). The shift between the two families could be explained by the polishing process (i.e. by the roughness) and by the surface contamination of the sample. Optical properties of metals are very sensitive to the surface contamination and the quality of polishing. A reliable explanation of the observed shift is possible only through a systematic study of optical properties as a function of these two parameters. Unfortunately we cannot provide a detailed explanation of the shift since information about the surface state of studied samples (in the literature) is too fragmentary. The LD model predicts a linear behavior only between 300 and $1000 \mathrm{~K}$ deviating for higher temperatures. Such deviation from linear increase is mostly due to the temperature dependence of electron-phonon damping of the Drude oscillator (the first term in Eq. 7). One can note that for $\theta / T<1$ (i.e. high temperatures), the electron-phonon damping can be approximated with a linear dependence on temperature given by

$$
\Gamma_{e-p h}(T)=\Gamma_{0}\left(\frac{2}{5}+\frac{T}{\theta}\right)
$$


Table 4. Total emissivity of molybdenum as a function of temperature evaluated with the modified Lorentz-Drude model

\begin{tabular}{cccc}
\hline \hline $\mathrm{T}(\mathrm{K})$ & $\epsilon_{\text {tot }}$ & $\mathrm{T}(\mathrm{K})$ & $\epsilon_{\text {tot }}$ \\
\hline 300 & 0.026 & 1750 & 0.185 \\
500 & 0.043 & 2000 & 0.214 \\
750 & 0.066 & 2250 & 0.240 \\
1000 & 0.092 & 2500 & 0.263 \\
1250 & 0.122 & 2800 & 0.285 \\
1500 & 0.154 & & \\
\hline \hline
\end{tabular}

as proposed by Maurya and Toutam [34]. By considering this modified LD model and using a $\Gamma_{0}$ value of 0.13 (instead of 0.02 as in the LD model) we succeed in fitting Worthing [35] and Matsumoto et al. results as shown by dashed line in Fig. 5c. Such modified LD model has been used to calculate the $\epsilon_{\lambda}$ for different wavelengths $(0.65,1$, 2, and $10 \mu \mathrm{m}$, dashed lines in Fig. 5b) and theoretical results are in better agreement with findings from Thomas [30] at $2400 \mathrm{~K}$.

\section{Summary and conclusion}

We have presented an experimental study of the temperature dependence of molybdenum reflectivity. Reflectivity measurements have been performed for $\mathrm{S}$ and $\mathrm{P}$ polarizations and for three angles of incidence $\left(20^{\circ}, 45^{\circ}\right.$, and $\left.50^{\circ}\right)$. We have measured a maximum increase of reflectivity in the 850 - $900 \mathrm{~nm}$ range: $0.8 \%$ between 300 and $540 \mathrm{~K}$ and $2.6 \%$ between 300 and $780 \mathrm{~K}$. The temperature dependence is not linear as a function of wavelength: the variation has a maximum in the 750-900 $\mathrm{nm}$ region while it is almost zero around $1050 \mathrm{~nm}$.

Using a model, based on Fresnel equations, we have determined the refractive index, $n$, and the extinction coefficient, $k$. We have found a $n$ value of 3.07 at $500 \mathrm{~nm}$, a slight increase to 3.27 at $750 \mathrm{~nm}$, and subsequent decrease to 2.58 at $1050 \mathrm{~nm}$. At $780 \mathrm{~K}$, n values present an increase comprises between $1.3 \%$ at $500 \mathrm{~nm}$ and $13.5 \%$ at $1050 \mathrm{~nm}$ with respect to room temperature. The extinction coefficient, $k$, is 3.7 at $550 \mathrm{~nm}$ and it is quite constant up to $850 \mathrm{~nm}$, increasing to 5.13 at $1050 \mathrm{~nm}$. As in the case of $n, k$ increases as a function of temperature with an overall growth of 8-10\% with respect to $300 \mathrm{~K}$.

We have developed a temperature dependent Lorentz-Drude model to simulate the electric permittivity of molybdenum. The model is able to fit reflectivity results with a maximum deviation of $1 \%$. We have also used the Lorentz-Drude model to estimate the spectral and total emissivity of molybdenum. Spectral emissivity has been evaluated in the $0.5-100 \mu \mathrm{m}$ range showing a significant increase as a function of temperature in the IR and far-IR domain. Total emissivity has been calculated up to the melting point of molybdenum $(\sim 2800 \mathrm{~K})$. The Lorentz-Drude model is not able to reproduce the linear increase of emissivity at temperatures higher than $1000 \mathrm{~K}$. We have explained the 
deviation from linear increase through the temperature dependence of electron-phonon damping of the Drude oscillator. We have succeed to fit emissivity only by considering a linear dependence of electron-phonon damping. We stress that such modification of electron-phonon damping temperature dependence does not affect emissivity predictions at temperatures below $1000 \mathrm{~K}$.

Molybdenum mirrors will be used in many optical diagnostic systems in ITER tokamak, and transitory temperature variations cannot be neglected in such harsh environment. For this reason the change of reflectivity of Mo mirrors as a function of temperature has to be considered to prevent possible failure of related diagnostic systems and to assure a perfect operability of the entire reactor.

\section{The project leading to this publication has received funding from Ex- cellence Initiative of Aix-Marseille University - A*MIDEX, a French "In- vestissements d'Avenir" programme}

[1] Sugie T, Costley A, Malaquias A and Walker C 2003 Journal of Plasma and Fusion Research 79 1051-1061

[2] Voitsenya V, Costley A E, Bandourko V, Bardamid A, Bondarenko V, Hirooka Y, Kasai S, Klassen N, Konovalov V, Nagatsu M, Nakamura K, Orlinskij D, Orsitto F, Poperenko L, Solodovchenko S, Stan? A, Sugie T, Taniguchi M, Vinnichenko M, Vukolov K and Zvonkov S 2001 Review of Scientific Instruments $\mathbf{7 2}$ 475-482

[3] Mukhin E, Semenov V, Razdobarin A, Tolstyakov S Y, Kochergin M, Kurskiev G, Podushnikova K, Masyukevich S, Kirilenko D, Sitnikova A, Chernakov P, Gorodetsky A, Bukhovets V, Zalavutdinov R K, Zakharov A, Arkhipov I, Khimich Y, Nikitin D, Gorshkov V, Smirnov A, Chernoizumskaja T, Khilkevitch E, Bulovich S, Voitsenya V, Bondarenko V, Konovalov V, Ryzhkov I, Nekhaieva O, Skorik O, Vukolov K Y, Khripunov V and Andrew P 2012 Nuclear Fusion 013017

[4] Ivanova D, Rubel M, Widdowson A, Petersson P, Likonen J, Marot L, Alves E, Garcia-Carrasco A, Pintsuk G and JET-EFDA Contributors 2014 Physica Scripta T159 014011 ISSN 0031-8949, $1402-4896$

[5] Bock M D, Barnsley R, Bassan M, Bertalot L, Brichard B, Bukreev I, Drevon J, Guern F L, Hutton R, Ivantsivskiy M, Lee H, Leipold F, Maquet P, Marot L, Martin V, Mertens P, Mokeev A, Moser L, Mukhin E, Pak S, Razdobarin A, Reichle R, Seon C, Seyvet F, Simrock S, Udintsev V, Vayakis $\mathrm{G}$ and Vorpahl C 2016 Journal of Instrumentation 11 P08010-P08010 ISSN 1748-0221

[6] De Temmerman G, Baldwin M J, Doerner R P, Nishijima D, Seraydarian R, Schmid K, Kost F, Linsmeier C and Marot L 2007 Journal of Applied Physics 102083302 ISSN 0021-8979, 1089-7550

[7] Litnovsky A, Wienhold P, Philipps V, Sergienko G, Schmitz O, Kirschner A, Kreter A, Droste S, Samm U, Mertens P, Donn A, Rudakov D, Allen S, Boivin R, McLean A, Stangeby P, West W, Wong C, Lipa M, Schunke B, De Temmerman G, Pitts R, Costley A, Voitsenya V, Vukolov K, Oelhafen P, Rubel M and Romanyuk A 2007 Journal of Nuclear Materials 363-365 1395-1402 ISSN 00223115

[8] Litnovsky A, Voitsenya V, Sugie T, De Temmerman G, Costley A, Donn A, Vukolov K, Orlovskiy I, Brooks J, Allain J, Kotov V, Semerok A, Thro P Y, Akiyama T, Yoshida N, Tokunaga T and Kawahata K 2009 Nuclear Fusion 49075014 ISSN 0029-5515, 1741-4326

[9] Rapp J, van Rooij G J, Litnovsky A, Marot L, De Temmerman G, Westerhout J and Zoethout E 2009 Physica Scripta T138 014067 ISSN 0031-8949, 1402-4896

[10] Wienhold P, Litnovsky A, Philipps V, Schweer B, Sergienko G, Oelhafen P, Ley M, De Temmerman G, Schneider W, Hildebrandt D, Laux M, Rubel M and Emmoth B 2005 Journal of Nuclear 
The effect of surface temperature on optical properties of molybdenum mirrors

Materials 337-339 1116-1120 ISSN 00223115

[11] Rubel M, Coad J, De Temmerman G, Hakola A, Hole D, Likonen J, Uytdenhouwen I and Widdowson A 2010 Nuclear Instruments and Methods in Physics Research Section A: Accelerators, Spectrometers, Detectors and Associated Equipment 818-822

[12] Eren B, Marot L, Langer M, Steiner R, Wisse M, Mathys D and Meyer E 2011 Nuclear Fusion 103025

[13] Eren B, Marot L, Wisse M, Mathys D, Joanny M, Travre J M, Steiner R and Meyer E 2013 Journal of Nuclear Materials S852-S855

[14] Moser L, Steiner R, Leipold F, Reichle R, Marot L and Meyer E 2015 Journal of Nuclear Materials 940-943

[15] Moser L, Marot L, Eren B, Steiner R, Mathys D, Leipold F, Reichle R and Meyer E 2015 Nuclear Fusion 55063020 ISSN 0029-5515, 1741-4326

[16] Leipold F, Reichle R, Vorpahl C, Mukhin E E, Dmitriev A M, Razdobarin A G, Samsonov D S, Marot L, Moser L, Steiner R and Meyer E 2016 Review of Scientific Instruments 11D439

[17] Maffini A, Moser L, Marot L, Steiner R, Dellasega D, Uccello A, Meyer E and Passoni M 2017 Nuclear Fusion 046014

[18] Moser L, Marot L, Steiner R, Reichle R, Leipold F, Vorpahl C, Le Guern F, Walach U, Alberti S, Furno I, Yan R, Peng J, Ben Yaala M and Meyer E 2017 Physica Scripta T170 014047 ISSN 0031-8949, 1402-4896

[19] Litnovsky A, Matveeva M, Herrmann A, Rohde V, Mayer M, Sugiyama K, Krieger K, Voitsenya V, Vayakis G, Costley A, Reichle R, De Temmerman G, Richter S, Breuer U, Buzi L, Mller S, Philipps V, Samm U, Wienhold P and the ASDEX Upgrade Team 2013 Nuclear Fusion 53 073033 ISSN 0029-5515, 1741-4326

[20] Salewski M, Meo F, Bindslev H, Furtula V, Korsholm S B, Lauritzen B, Leipold F, Michelsen P K, Nielsen S K and Nonbl E 2008 Review of Scientific Instruments 10E729

[21] Minissale M, Pardanaud C, Bisson R and Gallais L 2017 Journal of Physics D: Applied Physics 50455601

[22] Minissale M, Bisson R and Gallais L 2016 Laser remote heating in vacuum environment to study temperature dependence of optical properties for bulk materials LASER-INDUCED DAMAGE IN OPTICAL MATERIALS 2016 vol 10014 ed of SPIE P p 100141Q

[23] Querry M 1987 Optical constants of minerals and other materials from the millimeter to the ultraviolet Tech. rep. University of Missouri-Kansas City

[24] Werner W S M, Glantschnig K and Ambrosch-Draxl C 2009 Journal of Physical and Chemical Reference Data 38 1013-1092

[25] Kirillova M M, Nomerovannaya L V and Noskov M M 1971 Soviet Physics JETP 6

[26] Ordal M A, Bell R J, Alexander R W, Newquist L A and Querry M R 1988 Appl. Opt. 1203-1209

[27] Bouillard J S G, Dickson W, O?Connor D P, Wurtz G A and Zayats A V 2012 Nano Letters 12 $1561-1565$

[28] Cagran C, Pottlacher G, Rink M and Bauer W 2005 International Journal of Thermophysics $1001-1015$

[29] Wall R N, Basch D R and Jacobson D L 1992 Journal of Materials Engineering and Performance $1679-684$

[30] Thomas L K 1968 physica status solidi (b) 28 401-406

[31] Matsumoto T, Cezairliyan A and Basak D 1999 International journal of thermophysics 943-952

[32] Rudkin R L, Parker W J and Jenkins R J 1962 Temperature: Its Measurement and Control in Science and Industry vol 3 (Reinhold, New York) pp 523-534

[33] Abbott G, Alvares N J and Parker W J 1962 Total normal and total hemispherical emittance of polished metals Tech. rep. WADD Technical Report

[34] Maurya M R and Toutam V 2016 The Journal of Physical Chemistry C 19316-19321

[35] Worthing A G 1926 Phys. Rev. 28190 